\title{
Duhuo Jisheng Tang for treating osteoarthritis of the knee: a prospective clinical observation Jung-Nien Lai ${ }^{1}$, Huey-Jeng Chen ${ }^{2}$, Chao-Chung Chen ${ }^{3}$, Jer-Huei Lin ${ }^{4}$, Jing- Shiang Hwang 5 and Jung-Der Wang*6
}

\begin{abstract}
Address: ${ }^{1}$ Department of Obstetrics and Gynecology; Department of Chinese Medicine, Taipei City Hospital, Yangming Branch; Institute of Traditional Medicine, School of Medicine, National Yang-Ming University, No.155, Sec. 2, Linong Road, Taipei 112, Taiwan, ${ }^{2}$ Division of Chinese Internal medicine, Taipei City Hospital, Branch for Chinese Medicine, No.100, Kunming St., Taipei 108, Taiwan, ${ }^{3}$ Department of Traumatology, Taipei City Hospital, Branch for Chinese Medicine, No.100, Kunming St., Taipei 108, Taiwan, ${ }^{4}$ Division of Pharmacognosy, Bureau of Food and Drug Analysis, Department of Health, Executive Yuan, No.161-2, Kunyang St., Taipei 115, Taiwan, ${ }^{5}$ Institute of Statistical Science, Academia Sinica, No.128, Academia Road Sec. 2, Taipei 115, Taiwan and ${ }^{6}$ Departments of Internal Medicine and Environmental and Occupational Medicine, National Taiwan University Hospital and Institute of Occupational Medicine and Industrial Hygiene, National Taiwan University College of Public Health, No.17, Xuzhou Road, Taipei 100, Taiwan
\end{abstract}

Email: Jung-Nien Lai - kareny@ms10.hinet.net; Huey-Jeng Chen - chen7758@seed.net.tw; Chao-Chung Chen - leon.ccchen@msa.hinet.net; JerHuei Lin - linjerhuei@nlfd.gov.tw; Jing-Shiang Hwang - jshwang@stat.sinica.edu.tw; Jung-Der Wang* - jdwang@ntu.edu.tw

* Corresponding author

Published: 30 March 2007

Chinese Medicine 2007, 2:4 doi:10.1186/1749-8546-2-4

This article is available from: http://www.cmjournal.org/content/2/I/4

This is an Open Access article distributed under the terms of the Creative Commons Attribution License (http://creativecommons.org/licenses/by/2.0), which permits unrestricted use, distribution, and reproduction in any medium, provided the original work is properly cited.

Received: 17 November 2006

Accepted: 30 March 2007

\begin{abstract}
Background: Little scientific evidence supports the efficacy of herbal medicines in the treatment of degenerative arthritis of the knee. The purpose of this study is to evaluate both the efficacy and safety of a finished Chinese herbal preparation Duhuo jisheng Tang (DJT) in reducing symptoms of degenerative osteoarthritis of the knee.

Methods: A prospective follow-up study was carried out in two hospitals in Taipei between April and October 2005. Sixty-eight osteoarthritis patients, with symptoms diagnosed by radiologists, received DJT at a rate of $2.5 \mathrm{~g}$, twice daily for four weeks. Baseline scores were measured on the Western Ontario and McMaster Universities Osteoarthritis (WOMAC) index, followed by further measures at the end of weeks I, 2 and 4. The World Health Organization Quality of Life (WHOQOL) assessment was undertaken as a secondary outcome, with pattern identification questionnaires being adopted. Regression models were constructed to explore the score differences between the baseline and at weeks 2 and 4 by various determinants including age, gender, body mass index (BMI), severity at baseline, use of rescue medication, aversion to cold and flaccidity of the lower back and knees.
\end{abstract}

Results: Among the 68 participants, there were statistically significant reductions in the WOMAC index scores for pain, stiffness and physical functioning in the second and fourth weeks, with effects first appearing during week 2. By week 4, the mean WOMAC index scores had fallen from $22.2( \pm 19.2)$ to I6.I ( \pm 16.2) for pain, from $28.1( \pm 24.9)$ to $18.5( \pm 20.3)$ for stiffness, and from $22.6( \pm$ I8.0) to I8.2 ( \pm 17.8) for physical functioning, while the global score for pain under the visual analogue scale (VAS) was reduced from $38.7( \pm 21.5)$ to 27.8 ( \pm 19.8).

Conclusion: In the treatment of degenerative osteoarthritis of the knee, a 4-week therapy with the Chinese herbal preparation DJT reduced pain and stiffness and improved physical functioning, but it was less effective in treating flaccidity and aversion to cold. 


\section{Background}

Osteoarthritis (OA) is the most common form of joint disease, with the most common location being the knee [1]. As the patient ages, or the illness worsens, OA becomes associated with incapacity and deterioration in the quality of life owing to increased pain and loss of mobility [2]. For those OA patients who are not suited for surgery and/or those with ineffective medical treatment and/or intolerable side effects [3], complementary and alternative management approaches are frequently considered.

According to traditional Chinese medicine, arthritis belongs to the category of $b i$-syndrome, which is attributed to the weakening of the body's protective energy (weiqi). Weakened qi allows the incursion of the external pathogens (waixie) such as 'Wind', 'Cold' and 'Dampness' in Chinese medicine terminology. This incursion impedes the normal flow of $q i$ and results in pain and abnormal function of the human body. A combination of symptoms such as pain, stiffness, flaccidity and aversion to cold of the knee signifies disharmony caused by 'Wind', 'Cold' and 'Dampness'.

First documented about thirteen centuries ago in Qianjin Yaofang (Invaluable Prescriptions for Ready Reference), an ancient Chinese medicinal text [4,5], Duhuo Jisheng Tang (DJT) was used to treat symptoms caused by 'Wind', 'Cold' and 'Dampness'. DJT is a mixture of fifteen plant species as follows: Radix Angelicae Sinensis (Danggui), Radix Paeoniae Alba(Baishao), Radix et Rhizoma Glycyrrhizae (Gancao), Radix Rehmanniae (Dihuang), Radix et Rhizoma Ginseng (Renshen), Poria (Fuling), Radix Angelicae Pubescentis (Duhuo), Herba Taxilli (Sangjisheng), Radix Gentianae Macrophyllae (Qinjiao), Radix Saposhnikoviae (Fangfeng), Radix et Rhizoma Asari (Xixin), Rhizoma Chuanxiong (Chuanxiong), Cortex Cinnamomi (Rougui), Cortex Eucommiae (Duzhong), Radix Cyathulae (Chuanniuxi) [5] (Table $1)$.

Aristolochic acid I (AA-I), a known nephrotoxin [6], is found in a commonly used Chinese medicinal herb Xixin which is originated from nine Asarum species (Aristolochiaceae). All products containing Xixin have been prohibited in the US and Canada $[7,8]$. However, Xixin remains widely used in the DJT formula in Korea, Japan, Mainland China and Taiwan. A previous study showed that the side effects of DJT appeared at a daily dose of $9.0 \mathrm{~g}$ [11]. The commonly administered dosage in Taiwan is $5 \mathrm{~g}$ per day [9]. In 2003, the Committee of Chinese Medicine and Pharmacy (CCMP) in Taiwan removed all Aristolochic acid containing herbs except Xixin from the approved herbal products because of safety concerns. The committee also stipulated that the AA-I level in all Xixin preparations should be undetectable [10]. The current study is intended to evaluate the efficacy of DJT on patients with OA of the knee at a daily dose of $5.0 \mathrm{~g}$. The observation period was to last four weeks to test whether there are any cumulative effects. We also intended to verify an ancient indication that DJT was effective in treating pain, stiffness, flaccidity of the lower back and knee and aversion to cold.

\section{Methods}

The herbal preparation DJT used in this study was prepared under GMP (Good Manufacturing Practices) and provided by Sun Ten Pharmaceutical (Taiwan). The project was entirely funded by the CCMP to detect potential toxicity or adverse effects of DJT. Apart from providing the batch of DJT, Sun Ten Pharmaceutical was not involved in any other sponsorship, study design or monitoring of the participants.

\section{Preparation of the herbs}

All herbal components of DJT were prepared in a large computer-controlled boiler where volatile oils of therapeutic values were collected and sprayed into a two-story high vacuum drying chamber. The active ingredients of the herbs were transformed into granulated compounds five times more concentrated than the raw herbs. These granulated compounds were then vacuum dried at low temperatures before being siphoned into a separate sterile-room where they were bottled, labeled and sealed. For quality assurance of the active ingredients in DJT, HPLC (high performance liquid chromatography) fingerprinting was employed to identify substances in the final product [12]. An extraction process required by the CCMP regulations was performed, involving the use of water to eliminate AA-I from Asarum heteropoides roots, a nephrotoxic substance contained mostly in the leaves and/or aerial parts of plants [13]. Liquid chromatography - tandem mass spectrometry (LC/MS/MS) [14], a more sensitive method, had been carried out by the Bureau of Food and Drug Analysis of the Department of Health prior current study to ensure that AA-I was at an undetectable level. Each batch of DJT was also tested for E. coli, Salmonella (bacteria count) and heavy metals.

\section{Recruitment of subjects}

This prospective follow-up study was coordinated from the National Taiwan University, while participants were enrolled through two research clinics in northern Taiwan: Chinese Medicine Branch and Yangming Branch of the Taipei City Hospital. Approval of the study was obtained from the Joint Institutional Review Board for Traditional Chinese Medicine of Taiwan (JIRBTCM94-0426-01). All participants provided signed and informed consents before taking part in the study. 
Table I: Nomenclature of Chinese herbs used in Duhuo Jisheng Tang

\begin{tabular}{lll}
\hline Pharmaceutical name & Chinese pinyin & Latin botanical name \\
\hline Radix Angelicae Sinensis & Danggui & Angelica sinensis (Oliv.) Diels \\
Radix Paeoniae Alba & Baishao & Paeonia lactiflora Pall \\
Radix et Rhizoma Glycyrrhizae & Gancao & Glycyrrhiza uralensis Fisch. \\
Radix Rehmanniae & Dihuang & Rehmannia glutinosa Libosch. \\
Radix et Rhizoma Ginseng & Renshen & Panax ginseng C. A. Mey. \\
Poria & Fuling & Poria cocos (Schw.) Wolf \\
Radix Angelicae Pubescentis & Duhuo & Angelica pubescens Maxim.f. biserrata Shan et Yuan \\
Herba Taxilli & Sangjisheng & Taxillus chinensis (DC.) Danser \\
Radix Gentianae Macrophyllae & Qinjiao & Gentiana macrophylla Pall. \\
Radix Saposhnikoviae & Fangfeng & Saposhnikovia divaricata (Turcz.) Schischk. \\
Radix et Rhizoma Asari & Xixin & Asarum heterotropoides Fr. Schmidt var. mandshuricum (Maxim.) Kitag. \\
Rhizoma Chuanxiong & Chuanxiong & Ligusticum chuanxiong Hort. \\
Cortex Cinnamomi & Rougui & Cinnamomum cassia Presl \\
Cortex Eucommiae & Duzhong & Eucommia ulmoides Oliv. \\
Radix Cyathulae & Chuanniuxi & Cyathula officinalis Kuan \\
\end{tabular}

\section{Inclusion criteria}

Participants were recruited through newspaper advertisements and flyers posted in clinics and health fairs between April and October 2005. The status of osteoarthritis of the knee was confirmed by radiography (mild to moderate osteophytes and/or joint space narrowing). Qualified participants were at least 18 years of age with symptomatic osteoarthritis in at least one knee and had sought medical help over the two-week period prior to the study. Participants had not been involved in any other medical trials three months prior to the study. Participants were required to discontinue the use of any current medications, including any conventional or herbal products for arthritis, at least two weeks prior to the initial screening for the study.

\section{Exclusion criteria}

Rheumatoid, inflammatory or any other type of arthritis; arthroscopy or intra-articular corticosteroids/hyaluronic acid injections in the previous month; any evidence of renal or liver dysfunction as defined by a level of at least 1.5 times the upper reference limit [serum creatinine: 1.3 $\mathrm{mg} / \mathrm{dl}$, blood urea nitrogen (BUN): $22 \mathrm{mg} / \mathrm{dl}$, serum aspartate-aminotransferase (AST): $25 \mathrm{IU} / \mathrm{L}$, alanine-aminotransferase (ALT): $29 \mathrm{IU} / \mathrm{L}$; uncontrolled hypertension; diabetes mellitus; or any signs of cancer.

\section{Study design and procedure}

All nurses involved in the study attended a standardized training session to ensure consistency and to meet GCP (Good Clinical Practices) requirements in the study protocol. Participant eligibility was assessed during the first two visits to the clinic. Following the initial screening visit, participants entered a run-in phase to determine baseline data on their symptoms and their quality of life; each participant also underwent a health assessment including complete blood counts and biochemical function tests. These data were collected to ensure the eligibility criteria for each participant, and to screen out respondents with potential poor compliance. Following the run-in phase, each participant was provided with sufficient DJT to begin treatment at a dose of $2.5 \mathrm{~g}$ twice daily.

Subsequent study visits were scheduled for the first, second and fourth weeks, with any symptoms of the knee and adverse events being assessed at each visit. At the end of the trial, the participants received a further physical examination, including blood tests. Participants were contacted by telephone one to two days prior to each visit in order to encourage their continued compliance. Throughout the study period, leftover package counts were undertaken so as to monitor each participant's compliance. The consumption of non-steroidal anti-inflammatory drugs (NSAIDs) rescue medication was also recorded at each visit.

\section{Efficacy/Tolerability}

The primary outcome parameters evaluated prior to and during the four-week intervention were pain, stiffness and physical functioning subscales, along with the global pain assessment using a 100-mm 'visual analog scale' (VAS) from the Western Ontario and McMaster Universities Osteoarthritis (WOMAC) index [15]. We purchased the WOMAC Chinese language form directly from the author [16]. Changes in the quality of life were also assessed using the World Health Organization Quality of Life questionnaire - Taiwan brief version (WHOQOL-BREF) [17]. Each subject's physical constitution was also categorized using a questionnaire for pattern identification, or 'bian 
zheng' in Chinese medical terminology, based on the criteria recommended in Zhongyao Xinyao Linchuang Yanjiu Zhidao Yuanze (The Guidelines for Clinical Research on New Chinese Medicines) [18]. The questionnaire consisted of categories as follows: frequency of pain, aversion to cold and flaccidity of the lower back and knees (each of which were assessed in terms of 'occasional or less', 'often', or 'so frequent as to interfere with work'), and duration of stiffness ('less than one hour' or 'more than one hour').

The WOMAC index is a multi-dimensional, disease specific, self-administered, health status measure. It probes clinically important, patient-relevant symptoms in the areas of pain, stiffness and physical function in patients with knee osteoarthritis. The instrument comprises 24 questions (five on pain, two on stiffness and 17 on physical functioning) and can be completed in less than five minutes. Weightings were introduced to add together the five items on pain as a total sum score which predominantly quantifies the severity of knee pain in different daily activities, with the same process being carried out on stiffness and physical functioning [19]. The VAS version of the WOMAC is valid, reliable and sufficiently sensitive for the detection of clinically-important changes in health status following a variety of interventions $[20,21]$. The Taiwan version of the WHOQOL-BREF comprises four domains (i.e. physical, psychological, social and environmental) containing 24 facets, and two national items on overall quality of life (QOL) and general health [17], with higher scores indicating superior QOL.

\section{Safety assessment}

Routine hematology and biochemistry data were collected at the baseline and at week 4, including complete blood count and platelets, serum levels of creatinine, BUN, AST, ALT, albumin/globulin (A/G), uric acid and urine levels of $\mathrm{N}$-acetyl- $\beta$-D-glucosaminidase and retinol binding protein. A research nurse also actively monitored any adverse events and recorded any unexpected signs, symptoms or feelings during the study period.

\section{Statistical analysis}

Our analysis focused mainly on changes in the threedomain construct of the WOMAC assessment (pain, stiffness and physical function) along with the WHOQOL measures. The treatment effects were described as changes of the mean differences among the participants between the scores measured during each visit at weeks 1, 2, and 4 and at baseline. Separate linear regression models were constructed for the response variables of the differentiated scores on the pain, stiffness and physical functioning subscales, the VAS-pain and the WHOQOL-BREF domains.

The explanatory variables in the final regression model were gender, age, baseline severity of osteoarthritis, the interaction between age and gender, the BMI (in $\left.\mathrm{kg} / \mathrm{m}^{2}\right)$, aversion to cold, flaccidity of the lower back and knees and usage of NSAID rescue drugs. For us to examine the improvement across the 3 visits, two indicator variables for visits at weeks 2 and 4 were also included in the regression model. The coefficients of the two indicator variables represent the differences between the average value of measured scores for the visits at weeks 2 and 4 versus those at week 1 . The error terms were assumed to be correlated among the three repeated measurements on each participant. Finally, each single WOMAC score item was also examined separately so as to identify any WOMAC items sensitive to treatment by DJT. The estimates of coefficients and standard errors of the estimates in the multiple regression models with correlated error terms were obtained by using generalized least squares function of the package nlme version 3.1-60 in a free statistical software $\mathrm{R}$ version 2.1.1 [22].

\section{Results \\ Subjects}

Of the 87 sample patients, 18 were deemed ineligible. The principal reasons for ineligibility were uninterested in participation $(n=9)$, abnormal liver function $(n=5)$, abnormal renal function $(n=2)$ and no evidence of osteoarthritis $(\mathrm{n}=2)$. Sixty-eight of the initial 69 participants completed the four-week study. The main reason for the withdrawal was lack of efficacy during the first week of treatment. The demographic and clinical characteristics of the study subjects are summarized in Table 2. Among them, $37 \%$ were male, $82 \%$ married and $59 \%$ having attained senior high school education or above. Seven of the 68 took less than $80 \%$ of the prescribed dosage over the study period for the reason of lack of response to DJT. Patients with poor compliance had significantly lower scores in severity of pain and physical functioning and had significantly higher scores in physical QOL than those with good compliance.

\section{Efficacy on pain, stiffness and physical functioning}

After two weeks of treatment, there were significant and persistent improvements in each of the WOMAC subscales and also in the $10 \mathrm{~cm}$ VAS score for knee pain (Table 3). The magnitudes of the improvements in the WOMAC subscales were $27.5 \%$ for pain, $34.2 \%$ for stiffness and $19 \%$ for physical functioning. There was also a $28.2 \%$ improvement in the VAS score.

After four weeks of treatment, 44 of the 68 patients reported no change in the symptom of flaccidity; nine reported improvements, whereas fifteen reported deterioration. Thirty-eight patients reported no change in the symptom of aversion to cold after four weeks of treatment; fourteen reported improvements, while16 reported deterioration. 
Table 2: Baseline demographic and clinical characteristics for the 68 participants

\begin{tabular}{|c|c|}
\hline Definition & Mean (SD) \\
\hline Age (years) & $59.2(10.2)$ \\
\hline Body mass index & $24.0(3.2)$ \\
\hline \multicolumn{2}{|l|}{ Gender } \\
\hline$\%$ Male & 37 \\
\hline \multicolumn{2}{|l|}{ Religion } \\
\hline$\%$ Buddhist & 56 \\
\hline \% Christian & 10 \\
\hline \multicolumn{2}{|l|}{ Marital states } \\
\hline$\%$ Married & 82 \\
\hline \% Divorced/Widowed/Separated & 15 \\
\hline \multicolumn{2}{|l|}{ Education } \\
\hline \% Junior high or below & 41 \\
\hline$\%$ University or above & 34 \\
\hline Pain in visual analogue scale & $38.7(21.5)$ \\
\hline \multicolumn{2}{|l|}{ WOMAC Scores ${ }^{\mathrm{a}}$} \\
\hline Pain & $22.2(19.2)$ \\
\hline Stiffness & $28.1(24.9)$ \\
\hline Physical functioning & $22.6(18.0)$ \\
\hline \multicolumn{2}{|l|}{ Flaccidity of the lower back and knees } \\
\hline$\%$ Mild & 54 \\
\hline$\%$ Moderate & 32 \\
\hline$\%$ Severe & 13 \\
\hline \multicolumn{2}{|l|}{ Aversion to cold } \\
\hline$\%$ Mild & 73 \\
\hline$\%$ Moderate & 12 \\
\hline$\%$ Severe & 15 \\
\hline \multicolumn{2}{|l|}{ WHOQOL-BREF Scores ${ }^{b}$} \\
\hline Physiological domain & $12.9(2.4)$ \\
\hline Psychological domain & $13.0(2.2)$ \\
\hline Social domain & $13.5(1.9)$ \\
\hline Environment domain & $13.5(1.7)$ \\
\hline
\end{tabular}

a WOMAC refers to the Western Ontario and McMaster Universities Osteoarthritis Index.

b WHOQOL - BREF refers to the World Health Organization Quality of Life - brief version.

There were no significant improvements in these two symptoms after the treatment.

The results of the multiple linear regression analyses indicate the effects of the different determinants on the out- come scores of the VAS for pain and different WOMAC subscales (Table 4). After rescue medication and other determinants had been controlled, the model constructions demonstrated significant improvements in the severity of pain, stiffness and physical functioning after

Table 3: Means and standard deviation of major variables measured at baseline and weeks I, 2 and 4

\begin{tabular}{lcccc}
\hline \multicolumn{1}{c}{ Variable definition } & Baseline & Week I & Week 2 & Week 4 \\
\hline WOMAC Scores & & & Mean (SD) & Mean (SD) \\
$\quad$ Pain & Mean (SD) & Mean (SD) & $16.9(16.9)^{* *}$ & $16.1(16.2) * *$ \\
$\quad$ Stiffness & $22.2(19.2)$ & $21.4(20.9)$ & $17.4(18.5)^{* * *}$ & $18.5(20.3) * * *$ \\
$\quad$ Physical functioning & $28.1(24.9)$ & $25.8(23.1)$ & $18.2(15.8)^{* *}$ & $18.2(17.8) *$ \\
Pain in visual analogue scale & $22.6(18.0)$ & $22.4(19.5)$ & $27.0(21.5)^{* * *}$ & $27.8(19.8)^{* * *}$ \\
WHOQOL-BREF Scores & $38.7(21.5)$ & $33.0(20.7)^{*}$ & - & - \\
$\quad$ Physiological domain & $12.9(2.4)$ & - & - & $13.2(1.9)$ \\
Psychological domain & $12.4(2.0)$ & - & - & $12.3(1.5)$ \\
Social domain & $13.5(1.9)$ & - & - & $13.6(2.2)$ \\
Environment domain & $13.5(1.7)$ & - & - & $13.4(1.5)$ \\
\hline
\end{tabular}

a WOMAC refers to the Western Ontario and McMaster Universities Osteoarthritis Index. bWHOQOL - BREF refers to the World Health Organization Quality of Life - brief version.

$*$ indicates $P<0.05$; ** indicates $P<0.01$; and *** indicates $P<0.001$. 
Table 4: The estimates of regression coefficients and standard errors for modeling outcomes of VAS for pain and WOMAC subscales

\begin{tabular}{|c|c|c|c|c|c|c|c|c|}
\hline \multirow{4}{*}{ Variable definition } & & & \multicolumn{6}{|c|}{ WOMAC subscales' } \\
\hline & \multicolumn{2}{|c|}{ VAS for paina } & \multirow{2}{*}{\multicolumn{2}{|c|}{ Pain }} & \multirow{2}{*}{\multicolumn{2}{|c|}{ Stiffness }} & & \\
\hline & & & & & & & \multicolumn{2}{|c|}{ Physical function } \\
\hline & Coeff. & S.E. & Coeff. & S.E. & Coeff. & S.E. & Coeff. & S.E. \\
\hline Severity at baseline & $-0.40 * * *$ & 0.08 & $-0.42^{* * *}$ & 0.07 & $-0.5 I^{* * *}$ & 0.07 & $-0.34 * * *$ & 0.07 \\
\hline Mean score improvement at week 2 vs. week I & $-6.16^{* * * *}$ & 1.84 & $-4.62 * *$ & 1.55 & $-8.52^{* * *}$ & 2.11 & $-4.24 * *$ & 1.34 \\
\hline Mean score improvement at week 4 vs. week I & $-5.13 * * *$ & 1.84 & $-5.28 * * *$ & 1.55 & $-7.22^{* * *}$ & 2.11 & $-4.04 * *$ & 1.34 \\
\hline Age & 0.00 & 0.24 & 0.21 & 0.20 & 0.33 & 0.23 & $0.39 *$ & 0.18 \\
\hline Gender (female vs. male) & -15.74 & 19.73 & 3.83 & 16.67 & 2.18 & 19.38 & 13.26 & 15.06 \\
\hline Body Mass Index (<25 vs. $>=25$ ) & -0.68 & 3.44 & -1.03 & 2.92 & -1.25 & 3.44 & -1.19 & 2.65 \\
\hline Rescue drug (used vs. not used) & 6.03 & 3.38 & 4.27 & 2.85 & 3.79 & 3.65 & 4.86 & 2.52 \\
\hline Aversion to cold (mild vs. moderate or severe)c & 3.86 & 3.94 & 7.59 & 3.31 & $8.12^{*}$ & 3.88 & 3.93 & 3.09 \\
\hline Flaccidity of lower back/knee (mild vs. moderate or severe) c & 3.03 & 3.46 & 2.03 & 2.87 & -1.36 & 3.34 & 0.65 & 2.63 \\
\hline
\end{tabular}

aVAS = visual analogue scale as the dependent variables.

bRefers to the three subscales of the Western Ontario and McMaster Universities Osteoarthritis (WOMAC) Index as the dependent variables. cPattern identification as the independent variables.

*indicates $P<0.05$; ** indicates $P<0.01$; and *** indicates $P<0.001$.

week 2 (which also persisted through week 4), with substantial treatment effects on the stratum of the most severe pain (baseline VAS score $>4 \mathrm{~cm}, \mathrm{n}=32$ ). The frequency of either flaccidity or aversion to cold were not significantly affected in any of the above major outcome scores, and there were no consistent improvements with regard to these two symptoms after week 4 .

\section{Safety Issue}

There were no significant changes in all tested biomarkers including the highly sensitive indicators of nephrotoxicity $[23,24]$ after the 4 -week DJT intervention. Only four adverse drug reactions were potentially related to $D J T$ treatment, involving single events of skin discoloration, flashes, diarrhea and tachycardia.

\section{Discussion}

This study is observational in nature and lacks a randomized placebo group. We, therefore, cannot make strong inference from the outcomes reported by the patients. However, the present study recruited only the patients with radiographically-verified $\mathrm{OA}$ of the knee and used self-comparison to rule out the potential confounders of the BMI, age, exercise and socioeconomic status [25]. This study also showed that the supposed efficacy of DJT on flaccidity of the lower back and knee and aversion to cold, as indicated in Qianjin Yaofang (Invaluable Prescriptions for Ready Reference) [4], were not reflected in the 'relief of symptoms' or 'physical functioning' scores for $\mathrm{OA}$ of the knee (Table 4).

In current practice of Chinese medicine, DJT is usually prescribed as a treatment for a combination of symptoms of pain, stiffness, flaccidity and/or aversion to cold. The results of this study suggest that the 4-week treatment of the DJT preparation may not be effective in treating flaccidity and/or aversion to cold. As most participants in this study experienced only mild or moderate symptoms of flaccidity and/or aversion to cold, very limited room may have been left for improvements of the symptoms (Table 2).

Following the recommendations of the World Health Organization, we used the WHOQOL-BREF, a multidimensional measure of $\mathrm{QOL}$, as our secondary outcome in this study [26]. However, there were no statistically significant changes in the scores of either the WHOQOL domains or the different facets (Table 3 ) for those patients suffering from pain or stiffness who showed significant improvements. This may be due to the small sample size and the generic nature of the WHOQOL-BREF which, for most of the OA patients in this study with only mild symptoms, may not be so responsive.

Since DJT contains Xixin which may contain AA-I, an undetectable level of AA-I in the medications of the study was assured in compliance with the regulations of the CCMP. In contrast to the positive control sample directly taken from Xixin, no AA-I was detected in the independent chemical analysis of DJT by LC/MS/MS in this study.

The DJT dosage used in this study (2.5 g twice daily) is smaller than that in a previous study which showed that an oral daily dose of $9 \mathrm{~g}$ of $D J T$ had a similar toxicity profile to that of diclofenic [11]. The results of this study provide (about IIb level [26]) that the participants seemed well tolerant of the $5.0 \mathrm{~g}$ daily dose of the DJT preparation for four weeks, and that the four adverse drug reactions noted earlier were not serious enough to have caused any withdrawal by any of the participants. 
It should be noted that the results from the current study may not represent those of other types of DJT preparations, as different manufacturing processes may affect the biological activities and toxicity levels of herbs such as Xixin. Moreover, as the participants in this study were only Taiwanese patients suffering from OA of the knee during late spring and early autumn, we should remain cautious about generalizing these findings to cover patients in other settings, or indeed, to different racial groups.

\section{Conclusion}

The participants indicated symptomatic improvements in pain, stiffness and physical functioning as demonstrated in the WOMAC subscale scores which began to decrease after two weeks of treatment. Multiple regression analyses, however, showed that the DJT preparation was less effective on flaccidity of the lower back and knees and aversion to cold. The dosage form and prescription pattern of the DJT preparation described in the current study may be used as a complementary or alternative treatment of pain, stiffness and other physical functioning problems in patients with OA of the knee. As the results from the current study could have been confounded by placebo effect, natural fluctuation, insufficient follow-up time and/or other unobserved factors, a prospective randomized, double-blind, controlled trial to further evaluate the efficacy of DJT on OA patients is warranted in future studies.

\section{List of abbreviations}

DJT: Duhuo Jisheng Tang

WOMAC index: Western Ontario and McMaster Universities Osteoarthritis index

WHOQOL: World Health Organization Quality of Life

VAS: Visual analogue scale

OA: Osteoarthritis

AA-I: Aristolochic acid I

CCMP: Committee of Chinese Medicine and Pharmacy

GMP: Good Manufacturing Practices

HPLC: High performance liquid chromatography

LC/MS/MS: Liquid chromatography - tandem mass spectrometry

BUN: Blood urea nitrogen

AST: Serum aspartate-aminotransferase
ALT: Alanine-aminotransferase

GCP: Good Clinical Practices

NSAIDs: Non-steroidal anti-inflammatory drugs

WHOQOL-BREF: World Health Organization Quality of Life - brief version

QOL: Quality of life

A/G: Albumin/globulin

BMI: Body mass index

\section{Competing interests}

The author(s) declare that they have no competing interests.

\section{Authors' contributions}

JNL did the study design, patient recruitment, manuscript preparation and submission. HJC and CCC did the patient recruitment. JSH did all statistical analyses and interpretation of data. JHL did the LC/MS/MS analysis. JDW conceived the study, did its design and coordination, and helped draft the manuscript. All authors have read and approved the final manuscript.

\section{Acknowledgements}

The DJT study was funded by the following two grants: CCMP94-CP-005 from the Committee on Chinese Medicine and Pharmacy of the Department of Health (Taiwan), and NHRI-EX95-9204PP from the National Health Research Institutes (Taiwan). The authors would like to thank Drs Teh-Chen Wang, Yi-Jie Ou Yang for roentgenographic diagnosis. We are most grateful to all the patients who participated in this study for their valuable contribution.

\section{References}

I. Creamer P, Hochberg MC: Osteoarthritis. Lancet 1997, 350:503-8.

2. Felson DT: The epidemiology of knee osteoarthritis: Results from the Framingham osteoarthritis study. Semin Arthritis Rheum 1990, 20(3 suppl I):42-50.

3. Hernandez-Diaz S, Rodriguez LA: Association between non-steroidal anti-inflammatory drugs and upper gastrointestinal tract bleeding/perforation: An overview of epidemiologic studies published in the 1990s. Arch Intern Med 2000, I 60( I 4):2093-9.

4. Sun Si-miao : Qianjin Yaofang (Invaluable Prescriptions for Ready Reference) [http://www.npm.gov.tw/exh92/medicinal9207/english/content/ m4.htm]. National Palace Museum, Taipei, Taiwan

5. Committee on Chinese Medicine and Pharmacy, Department of Health, Executive Yuan, Taiwan: The Standardized Formulae [http:// www.ccmp.gov.tw/information/license.asp].

6. Cosyns JP: Aristolochic acid and 'Chinese herbs nephropathy': A review of the evidence to date. Drug Saf 2003, 26(I):33-48.

7. Office of Nutritional Products, Labeling and Dietary Supplements, United States Food and Drug Administration: Letter to industry associations regarding safety concerns related to the use of botanical products containing Aristolochic acid [http://www.cfsan.fda.gov/ dms/dsbotl4.html].

8. Health Canada: Health Canada advises consumers not to use products containing Aristolochic acid [http://www.hc-sc.gc.ca/ahc-asc/media/advi sories-avis/2005/2005 08 e.html]. 
9. Tseng WL: Frequency of prescribing aristolochic acid-associated Chinese herb medicine in National Health Insurance in Taiwan, 1997-200 I. In Masters Thesis National Taiwan University, Institute of Occupational Medicine and Industrial Hygiene; 2005.

10. Committee on Chinese Medicine and Pharmacy Department of Health, Executive Yuan, Taiwan: Analysis and Specification of Aristolochic Acid in Xixin [http://www.ccmp.gov.tw/public/pub lic.asp? selno $=60 \mid$ \&relno $=60 \mid$ \&level $=C]$

II. Teekachunhatean S, Kunanusorn P, Rojanasthien N, Sananpanich K, Pojchamarnwiputh S, Lhieochaiphunt S, Pruksakorn S: Chinese herbal recipe versus diclofenac in symptomatic treatment of osteoarthritis of the knee: a randomized controlled trial. BMC Complement Altern Med 2004, 4(I): 19-27.

12. Sun Ten Phytotech Co., Ltd: Quality Assurance for Botanical Drugs [http://www.suntenglobal.com/about/milestones.php].

13. Drew AK, Whyte IM, Bensoussan A, Dawson AH, Zhu XS, Myers SP: Chinese herbal medicine toxicology database: monograph on Herba Asari, 'Xi Xin'. J Toxicol Clin Toxicol 2002, 40(2): 169-72.

14. Huang $\mathrm{CY}$, Tseng MC, Lin JH: Analyzing Aristolochic acids in Chinese herbal preparations using LC/MS/MS. J Food Drug Anal 2005, I3(2): |25-3|.

15. McConnell S, Kolopack P, Davis AM: The Western Otario and McMaster Universities Osteoarthritis Index (WOMAC): A review of its utility and measurement properties. Arthritis Care Res 200I, 45:453-6I.

16. WOMAC ${ }^{\mathrm{TM}}$ Osteoarthritis Index: Knee and Hip Osteoarthritis Index [http://www.auscan.org/womac/index.htm\#asiapacific].

17. Yao G, Chung CW, Yu CF, Wang JD: Development and verification of validity and reliability of the WHOQOL-BREF Taiwan version. J Formos Med Assoc 2002, I0I:342-5I.

18. Zheng XY, Ed: Zhonghu mianyi jibing. In Zhongyao Xinyao Linchuang Yanjiu Zhidao Yuanze (The Guidelines for Clinical Research on New Chinese Medicines) Beijing: China Medicine Technology Press; 2002:115-9.

19. Angst F, Stucki G, Aeschlimann A: Quality of life assessment in osteoarthritis. Expert Rev Pharmacoeconomics Outcome Res 2003, 3:623-36.

20. Bellamy N, Campbell J, Robinson V, Gee T, Bourne R, Wells G: Intraarticular corticosteroid for treatment of osteoarthritis of the knee. Cochrane Database Syst Rev 2005:CD005328.

21. Arden N, Nevitt MC: Osteoarthritis: Epidemiology. Best Pract Res Clin Rheumatol 2006, 20(I):3-25.

22. R Development Core Team: $R$ : A language and environment for statistical computing Version 2.I.I. Vienna 2005 [http://www.R-project.org].

23. Price RG: Early markers of nephrotoxicity. Comp Clin Path 2002 I I:2-7.

24. Price RG: The role of NAG (N-acetyl-beta-D-glucosaminidase) in the diagnosis of kidney disease including the monitoring of nephrotoxicity. Clin Nephro 1992, 38(SuppI I):SI4-9.

25. Towheed TE: Pennsaid therapy for osteoarthritis of the knee: a systematic review and metaanalysis of randomized controlled trials. J Rheumato 2006, 33(3):567-73.

26. WHO: General Guidelines for Methodologies on Research and Evaluation of Traditional Medicines. In Document: WHO/ EDM/TRM/2000.I Geneva: World Health Organization; 2000.

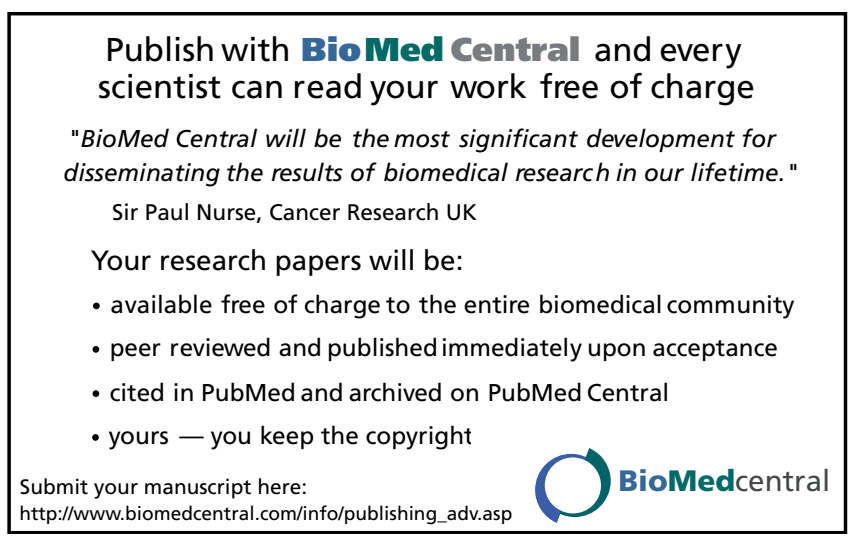

